L'enseignement à distance de l'éducation physique et sportive au temps du Corona Virus - cas du Maroc -

Aziz Eloirdi $^{\text {** }}$, Ayoub Koutaya ${ }^{\mathrm{a}}$, Mohamed Barkaoui ${ }^{\mathrm{a}}$, Assia Marfouq ${ }^{\mathrm{a}}$, Younes Koutaya ${ }^{\mathrm{b}} \&$ Hamid Eloirdi ${ }^{\mathrm{c}}$

${ }^{a}$ Hassan First University, Settat, Morocco

${ }^{b}$ Mohammed V University, Rabat, Morocco

${ }^{c} I b n$ Tofaïl University, Kenitra, Morocco

*aziz.eloirdi@uhp.ac.ma 


\section{L'enseignement à distance de l'éducation physique et sportive au temps du Corona Virus : cas du Maroc}

\section{Résumé}

La formation à distance ou l'apprentissage à distance connait son plein essor ces dernières années dans plusieurs régions du monde. Il résulte de cet intérêt que l'émersion de l'apprentissage à distance a été remarquablement prôné par les établissements d'enseignement supérieur et de moins en moins au niveau secondaire et collégial où la formation en présentiel devient essentielle. Cependant, pendant la période de crise sanitaire à cause du Covid-19, l'enseignement à distance s'était imposé comme un nouveau mode alternatif pour le présentiel pour tous les niveaux d'enseignement, et pour toutes les matières confondues. L'éducation physique et sportive (EPS) n'échappe pas à ce changement. Dans ce contexte, la discipline EPS a suscité un débat sur les modalités d'engagement des professeurs d'EPS pour participer à l'enseignement à distance vu la nature quasiment pratique de la matière. Devant ce problème, les enseignants d'EPS (lycée et collège) se sont trouvés contraint de formaliser des contenus pédagogiques et de les transmettre sur différents supports médiatiques. Cette étude contribue à l'identification du degré d'engagement des enseignants d'EPS pour assurer la durabilité des apprentissages et de déterminer les entraves rencontrées dans cette opération qualifiée d'urgente. Cette étude a vu la participation de 166 enseignants dans deux niveaux d'enseignements secondaire et collégial à travers trois académies régionales au Maroc. Les résultats ont montré à la fois l'absence de la communication entre les inspecteurs de l'EPS et les professeurs de la matière, et la coordination entre les professeurs au sein des unités pédagogiques.

Mots clés : enseignement à distance, éducation physique et sportive, apprentissage, technopédagogie, innovation pédagogique. 


\title{
Distance learning of physical and sports education in the days of the Corona Virus: Case of Morocco
}

\begin{abstract}
Distance training or distance learning has experienced a boom in recent years in several regions of the world. It follows from this interest that the emergence of distance learning has been remarkably advocated by higher education institutions and less and less at the secondary and college levels where face-to-face training is becoming essential. However, during the period of health crisis due to Covid19, distance education had established itself as a new alternative mode for faceto-face education for all levels of education, and for all subjects combined. Physical and sports education (PSE) is no exception to this change. In this context, the discipline EPS has sparked a debate on the modalities of engagement of teachers of EPS to participate in distance education given the almost practical nature of the subject. Faced with this problem, EPS teachers (high school and college) found themselves forced to formalize educational content and transmit it on various media. This study contributes to the identification of the degree of commitment of PE teachers to ensure the sustainability of learning and to determine the obstacles encountered in this operation qualified as urgent. This study saw the participation of 166 teachers in two levels of secondary and college education through three regional academies in Morocco. The results showed both the lack of communication between the inspectors of the EPS and the teachers of the subject, and the coordination between the teachers within the teaching units.
\end{abstract}

Keywords: distance education, physical and sports education, learning, technopedagogy, pedagogical innovation.

\section{Introduction}

Afin d'éviter la propagation de l'épidémie du coronavirus et pour assurer la continuité de l'enseignement pendant la période du confinement, le Ministère de l'Éducation Nationale, était contraint de fermer ses établissements publiques et privés, a mis en place un dispositif de cours à distance lancé depuis le 16 mars 2020 et présenté sur une panoplie de supports médiatiques : chaînes TV, portails électroniques, réseaux 
sociaux, etc. Dans ce contexte, le corps professoral de toutes les disciplines enseignées s'est engagé pour assurer la continuité pédagogique du contenu du programme.

En effet, l'enseignement à distance n'est pas un dispositif récent au Maroc, des centaines de capsules éducatives existaient déjà dans la plateforme ministérielle réservée à cette fin, mais le contexte actuel dû à l'état d'urgence a incité le ministère à adopter une communication de crise particulière et de prendre plus au sérieux l'enseignement à distance. Les contenus proposés aux élèves comme aux étudiants sont conformes aux programmes annuels réalisés par les enseignants en présentiel et prennent souvent la formes de capsules télévisées ou de conversations en ligne (visioconférence, chat, etc.). Ces contenus dépassent de simples ressources éducatives diffusées, car leur architecture répond parfaitement aux besoins d'un apprentissage qui se fait à la maison. Leur scénarisation est rigoureuse et consistante car elle obéit à un temps limité qui n'excède pas 30 min maximum et une forme de contenus variée (rappel, introduction, explications de la séquence du cours, illustrations, synthèse, exercices). Chaque séance de cours est enregistrée de manière permanente sur la plateforme ministérielle et fait l'objet d'une rediffusion dans les chaines télévisées dédiées à cette fin, suivant des créneaux déterminés, ce qui permet aux élèves d'accéder à ces contenus de manière répétitive et de travailler à leurs rythmes.

Certes, l'EPS présente une particularité remarquable par rapport aux autres disciplines enseignées vu plusieurs raisons. Tout d'abord, cette discipline combine le volet théorique et pratique avec prédominance du deuxième volet, ensuite c'est une discipline qui n'est pas sanctionnée par un examen régional ou national ce qui pousse les élèves à l'abandonner en croyant que c'est une discipline secondaire et sans utilité. Toutes ces données ont suscité notre curiosité pour interpréter les démarches prônées par les différents acteurs pendant cette période de confinement d'une part, et d'autre 
part déterminer le degré d'engagement des professeurs d'EPS dans cette opération. $\mathrm{Vu}$ sa nature quasiment pratique, la discipline EPS a posé une kyrielle de questions par rapport à la modalité d'exécution de l'enseignement de ces cours à distance. Alors, qu'en est-il pour l'enseignement de l'EPS au Maroc dans ce contexte de crise sanitaire où les élèves confinés sont dépourvus de mouvements? Les professeurs d'EPS ont-ils reçu une formation dans ce sens ? Ont-ils manifesté un réel engagement pour réussir cette opération d'enseignement à distance ? Les contenues publiées ou diffusées sontelles le produit de concertation entre les différents acteurs pédagogiques et éducatifs en EPS ou sont le résultat de réalisations individuelles?

En effet, l'intérêt de cette étude est de dresser un état des lieux de l'enseignement/apprentissage de L'EPS à distance. Nous rappelons que cette recherche puise son originalité dans le fait qu'il y a absence d'étude que ce soit anglophone ou francophone traitant de l'enseignement de l'EPS à distance. A l'aide des méthodes d'investigation empiriques reposant sur un questionnaire distribué en ligne auprès des enseignants d'EPS, nous essayerons de répondre à nos questionnements. Finalement, nous proposerons des pistes et des suggestions permettant un passage souple entre en présentiel et à distance qui puisse prendre en compte les spécificités de cette discipline pratique et les données contextuelles pour répondre aux attentes de ses acteurs. Aussi, des mesures seront suggérées pour améliorer la pratique enseignante de l'EPS à distance.

Cet article est organisé de la manière suivante : Dans une première partie nous apporterons un éclairage théorique sur les notions et les démarches développées dans l'enseignement à distance (EAD) en l'occurrence l'apprentissage en ligne, la formation des enseignants à l'EAD et la coopération entre les acteurs pédagogiques. Dans une deuxième partie, nous exposerons le processus d'apparition de l'EPS dans le numérique 
et les éventuelles contraintes que pourraient rencontrer les acteurs pédagogique suite au lancement de l'opération enseignement en ligne de toutes les matières. Dans une troisième partie, nous présenterons la méthodologie suivie pour le recueil des données et la dernière partie sera consacrée à la fois à la présentation et à la discussion des résultats de l'enquête et l'exposition des principales actions à entreprendre pour faciliter l'exécution de l'enseignement de l'EPS à distance.

\section{Apprendre en ligne : éléments de cadrage}

En l'espace d'une trentaine d'années, le domaine l'enseignement à distance, très marginal au départ, s'est imposé progressivement comme l'un des alternatives pour optimiser la durabilité des apprentissages. Nous pouvons le qualifier comme une révolution du système traditionnel de transmission des savoirs en apprentissage présentiel (Blandin 1990). L'apprentissage à distance utilise de nombreux supports et de modalités d'apprentissage. Il s'effectue généralement sur les réseaux numériques, à l'aide d'un ordinateur, d'un smartphone, ou d'une tablette. L'élève est toujours au centre du processus d'apprentissage (Jaillet 2005).

L’Office québécois de la langue française définit la formation en ligne comme « étant un mode d'apprentissage qui est basé sur l'utilisation des nouvelles technologies de l'information et de la communication, particulièrement via Internet. Ce mode permet l'accès à des formations interactives et personnalisées qui visent le développement de compétences tout en rendant la formation indépendante de l'heure et de l'endroit ».

L'enseignement à distance désigne les formes d'apprentissage et d'encadrement qui se déroulent partiellement ou complètement hors du campus connu sous les désignations d'enseignement hybride ou à distance et souvent formulé en anglais $e$ learning, blendedlearning, mobil learning, MOOC, adaptive learning... (Charnet, 2019). Selon l'Union Européenne : L'enseignement à distance est l'utilisation des 
nouvelles technologies multimédia et de l'Internet, pour améliorer la qualité de l'apprentissage en facilitant l'accès à des ressources et des services, ainsi que des échanges et collaboration à distance. Romiszowski (2003) constate qu'il existe deux modes de fonctionnement complémentaires de l'enseignement à distance :

- Mode synchrone : c'est une forme de communication directe en temps réel dans laquelle tous les participants sont connectés en même temps et communiquent directement les uns avec les autres (vidéoconférence)

- Mode asynchrone : c'est une forme de communication différée dans le temps. Les informations sont stokes de manière que les interlocuteurs puissent les rejoindre au moment qui leur convient. Les échanges ont lieu par courriel, forum ou via des plates-formes.

L'enseignement ou l'apprentissage à distance est une pratique récemment développée. On constate au cours des dix dernières années un intérêt croissant à ce sujet. Cette démarche semblerait nécessaire selon certains travaux de recherche portant sur l'encadrement des étudiants.

\section{La formation des enseignants pour l'enseignement à distance}

Joab et Dumont (2008) déclarent que parmi les contraintes au développement de la qualité dans les formations à distance, il y a le manque de formation des enseignants. Selon Dumont (2007), il apparaît nécessaire que les enseignants commencent à se familiariser avec les outils numériques développés comme support pédagogique d'apprentissage. Dumont (2007) souligne qu'à l'ère du digital, les fonctions et les rôles ne cessent de multiplier. Dès lors, les enseignants font face à une génération surdouée dans l'utilisation des outils numériques. En conséquence les enseignants sont interpellés à adhérer à cette vague révolutionnaire. Pour Gélis (2013), il est temps de penser au 
changement de paradigme d'enseignement et de s'ouvrir par conséquent aux nouvelles démarches d'enseignement à l'instar de l'EAD.

A l'issu de l'apprentissage à distance, l'enseignant est responsable de la conception et la conduite des séquences pédagogiques (George, S., 2004). Dans ce sens, l'enseignant reste le principal concepteur et diffuseur d'informations par de nombreuses techniques communicationnelles. Denis et al. (2004) estiment que le tuteur doit assurer les missions assignées dans le cadre des apprentissages e-learning à savoir :

- Construire une session de formation contenant tant de points : un scénario, des situations pédagogiques, des cas à étudiés ;

- Animer les sessions de formation et optimiser la créativité chez les apprenants ;

- Animer éventuellement des forums et des classes virtuelles ;

- Réajuster les apprentissages en fonction des capacités intellectuelles ;

Stockless et Villeneuve (2017) estiment que l'accompagnement des apprenants à distance exige une bonne compréhension du bon usage des outils numériques. D'ailleurs, Audet (2014) montre qu'en plus de la maitrise de l'outil numérique qui reste pour lui nécessaire, l'enseignant doit penser au scénario d'apprentissage qui mène par la suite au choix de la ressource numérique.

\section{La coopération entre les acteurs pédagogiques :}

Dans l'enseignement à distance, la coopération entre les acteurs est indispensable. Dans son guide de formation et de soutien des acteurs, Marcelle Parr (2019) met en exergue les conditions de réussite du projet d'apprentissage à distance : faciliter les communications, outiller les démarches, gérer les divergences, s'assurer de l'adhésion au projet, reconnaître les apports. Par cette ligne de conduite, Marcelle Parr (2019) considère qu'il parait fondamental de focaliser l'attention sur les rapports étroits entre 
l'enseignant producteur du contenu et le conseiller pédagogique. Selon l'auteur, le conseiller pédagogique complète le rôle d'accompagnateur, agit comme agent de changement et s'assure de la bonne marche du projet. De plus, le conseiller pédagogique essaie avec les outils de communication disponibles d'assister l'enseignant dans sa réflexion sur la structuration pédagogique des activités. La collaboration entre les acteurs pédagogiques soulève le rôle majeur de la communication dans toutes actions entreprises. Cette complémentarité des rôles facilite la bonne conduite du processus enseignement à distance et évite par conséquent l'apparition de tout acte de refus ou de résistance

\section{Quel contexte de l'enseignement de l'EPS à distances au Maroc?}

L'introduction des séances d'EPS au profit des élèves, tous cycles confondus, en période de crise sanitaire est une nécessité majeure envers les élèves qui se réorganisent pour poursuivre tant bien que mal, totalement ou partiellement leurs cours à distance et à domicile. Dans ces conditions particulières, la mise en place de la digitalisation des cours et leur suivi à distance induit pour les élèves une diminution du niveau d'activité physique mais également une exposition croissante au comportement sédentaire traduit principalement par « la position assise ou allongée avec une faible dépense énergétique ${ }^{1}$, ce qui nécessite une certaine vigilance de la part des éducateurs.

Ceci dit, il est d'une importance majeure pour les enseignants d'EPS de se manifester remarquablement dans le cadre du confinement vécu par les élèves et d'essayer d'apporter des solutions pour améliorer la qualité de vie des élèves et veiller

\footnotetext{
${ }^{1}$ http://www.inrs.fr/risques/teletravail-situation-exceptionnelle/prevenir-comportement-
} sedentaire.html (consulté le 18/05/2020). 
sur leur bien-être en leur proposant des activités physiques régulières adaptées à leur situation de confinement et aux exigences sanitaires et capable d'assurer une prévention contre la pandémie. ${ }^{2}$

Durant la période de la crise sanitaire de Covid-19, une décision a été promulguée par la haute direction du Ministère de l'Education Nationale pour l'engagement de l'éducation physique et sportive au mouvement de l'enseignement à distance. Dans ce contexte, les inspecteurs de l'EPS et les directeurs des établissements scolaires ont commandités les professeurs d'EPS pour proposer des initiatives d'ordre création des capsules vidéo diffusées sur les réseaux sociaux ou les applications de chat. Certes, cette opération a implicitement évalué la capacité des enseignants à utiliser les différents supports digitaux et particulièrement les sites internet et les réseaux sociaux. Au gré de cette vaste opération nationale, notre intérêt consiste à comprendre dans quelle mesure les enseignants d'EPS sont motivés pour s'engager dans cet état d'urgence et quels sont les dysfonctionnements que manifeste cette opération.

\section{Méthode de travail}

Si notre recherche tend à soulever les contraintes et les raisons de motivation et de démotivation des enseignants d'EPS pour la démarche d'enseignement à distance, il faut préciser que c'est une première initiative de ce genre au Maroc. De plus, la revue de littérature sur cette thématique apparaît inexistante puisque la matière EPS pose la particularité d'une matière d'enseignement où la pratique sportive compte fondamentale.

\footnotetext{
${ }^{2}$ Le Dr François Carré confirme que « Toutes les études scientifiques ont prouvé que l'activité physique modérée boostait » notre système immunitaire.
} 
En vue de servir la littérature sur l'enseignement de l'éducation physique et sportive, nous nous sommes appuyés sur la méthode des études de cas. Pour ce faire, nous avons fait appel à un échantillon de 166 enseignants d'EPS issus de trois académies régionales au Maroc : Casablanca-Settat, Marrakech-Safi et Fès-Meknès.

\section{Outil de collecte des données}

Lors de notre étude, nous avons conçu un questionnaire composé de 15 questions de natures différentes : des questions fermées et des questions à choix multiples (voir annexe 1). L'objectif est de diversifier la composition des questions et de chercher la congruence des enseignants d'EPS pour répondre à notre enquête ainsi que comprendre leur représentation à propos de l'enseignement à distance. La formulation des questions suppose de multiples intérêts :

- Les trois premières questions cherchent à identifier le profil et la durée de l'expérience professionnelle de l'enseignant.

- Les questions 4, 5 et 6 rapportent à la fois sur la capacité des enseignants de l'utilisation des supports numériques et leur réflexion sur l'enseignement de l’EPS à distance.

- La septième et la huitième question ont pour intérêt de déterminer l'existence d'une communication entre les conseillers pédagogiques et les professeurs d'une part, et d'autre part entre les enseignants.

- Les questions 9, 10 et 11 rapportent sur l'engagement pour la conception des cours à distance, la nature du cours (théorique ou pratique) et l'outil utilisé pour diffuser le contenu.

- La douzième question permet de soulever les contraintes rencontrées au cours de cette nouvelle opération. 
- Les trois dernières questions du questionnaire projettent trois thématiques essentiels à investiguer : la capacité d'innovation chez les enseignants (proposition des activités morales pour les élèves), la formation continue des enseignants sur les nouvelles technologies et la responsabilité éducative et éthique des enseignants pour cette nouvelle expérience.

\section{Échantillon de l'étude}

Notre échantillon est représenté par des professeurs d'éducation physique et sportive, appartenant à deux niveaux d'enseignement : premier cycle et second cycle qualifiant appartenant au secteur public. Notre enquête a sollicité la participation de 166 enseignants qui ont répondu à notre questionnaire. Nous précisons que cette enquête a été réalisée en coordination avec les inspecteurs de l'EPS dans chaque académie. Dans une période d'une semaine nous avons collecté les données et nous les avons traitées dans l'outil SPSS.

\section{Résultats de l'enquête}

\section{Répartition de l'échantillon}

La répartition de l'échantillon selon l'âge a montré que 75 participants soit $45,7 \%$ ont un âge supérieur à 42 ans, 48 cas (29.3\%) sont âgés entre 32 et 42 ans et 41 cas (25\%) ont un âge compris entre 21 et 31 ans. Selon l'expérience professionnelle, 60 participants soit $36.6 \%$ ont plus que 22 ans d'expérience, $27.4 \%$ et 36 cas (22\%) ont moins de 6 ans d'expérience professionnelle. Concernant le niveau d'enseignement, nous constatons que $60.4 \%$ des participants sont des professeurs de l'enseignement secondaire qualifiant et $39.6 \%$ sont des professeurs de l'enseignement secondaire collégial. 
Tableau1 : Répartition de l'échantillon selon l'âge, l'expérience professionnelle et le niveau d'enseignement

\begin{tabular}{|c|c|c|c|}
\hline Variables & Classes & Fréquence & Pourcentage \\
\hline \multirow{3}{*}{ Age } & $21-31$ & 41 & 25,0 \\
\hline & $32-42$ & 48 & 29,3 \\
\hline & 42 et + & 75 & 45,7 \\
\hline \multirow{4}{*}{$\begin{array}{l}\text { Expériences } \\
\text { professionnelles }\end{array}$} & +22 ans & 60 & 36,6 \\
\hline & +6 ans & 45 & 27,4 \\
\hline & +12 ans & 23 & 14,0 \\
\hline & +2 ans & 36 & 22,0 \\
\hline \multirow{4}{*}{$\begin{array}{l}\text { Niveau } \\
\text { d'enseignement }\end{array}$} & $\begin{array}{l}\text { Enseignement } \\
\text { secondaire } \\
\text { collégial }\end{array}$ & 65 & 39.6 \\
\hline & Enseignement & & \\
\hline & secondaire & 99 & 60.4 \\
\hline & qualifiant & & \\
\hline
\end{tabular}

L'enseignement à distance de l'EPS 


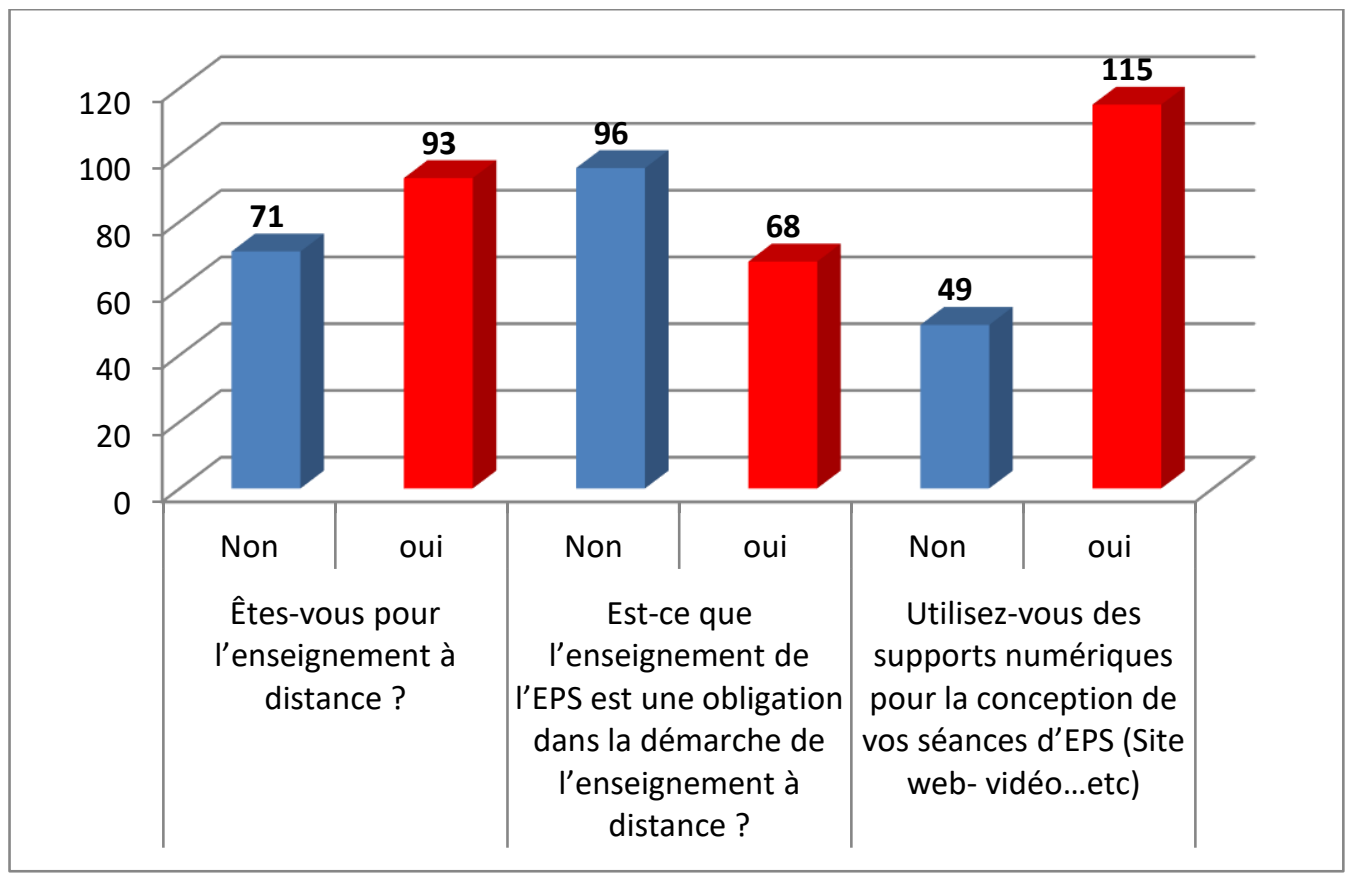

Figure1 : Perceptions des participants de l'enseignent à distance de l'EPS

D'après la figure ci-dessus, nous constatons que 93 des cas soit $56.7 \%$ sont pour l'enseignement à distance de l'EPS et 71 cas (43.3\%) sont contre cette démarche, la différence n'est pas significative $\left(\chi^{2}=2,951 ; p=0.08\right)$. Ainsi, 96 participants soit $58.5 \%$ déclarent que l'enseignement à distance de l'EPS n'est pas une obligation et $41.5 \%$ déclarent le contraire, la différence est statistiquement significative $\left(\chi^{2}=4,780\right.$; $\mathrm{p}=0.02$ ). Concernant la conception des séances d'EPS, 115 participants soit $70.1 \%$ utilisent des supports numériques et le reste ne les utilise pas, la différence est très significative $\left(\chi^{2}=26,561 ; \mathrm{p}<0.001\right)$. 


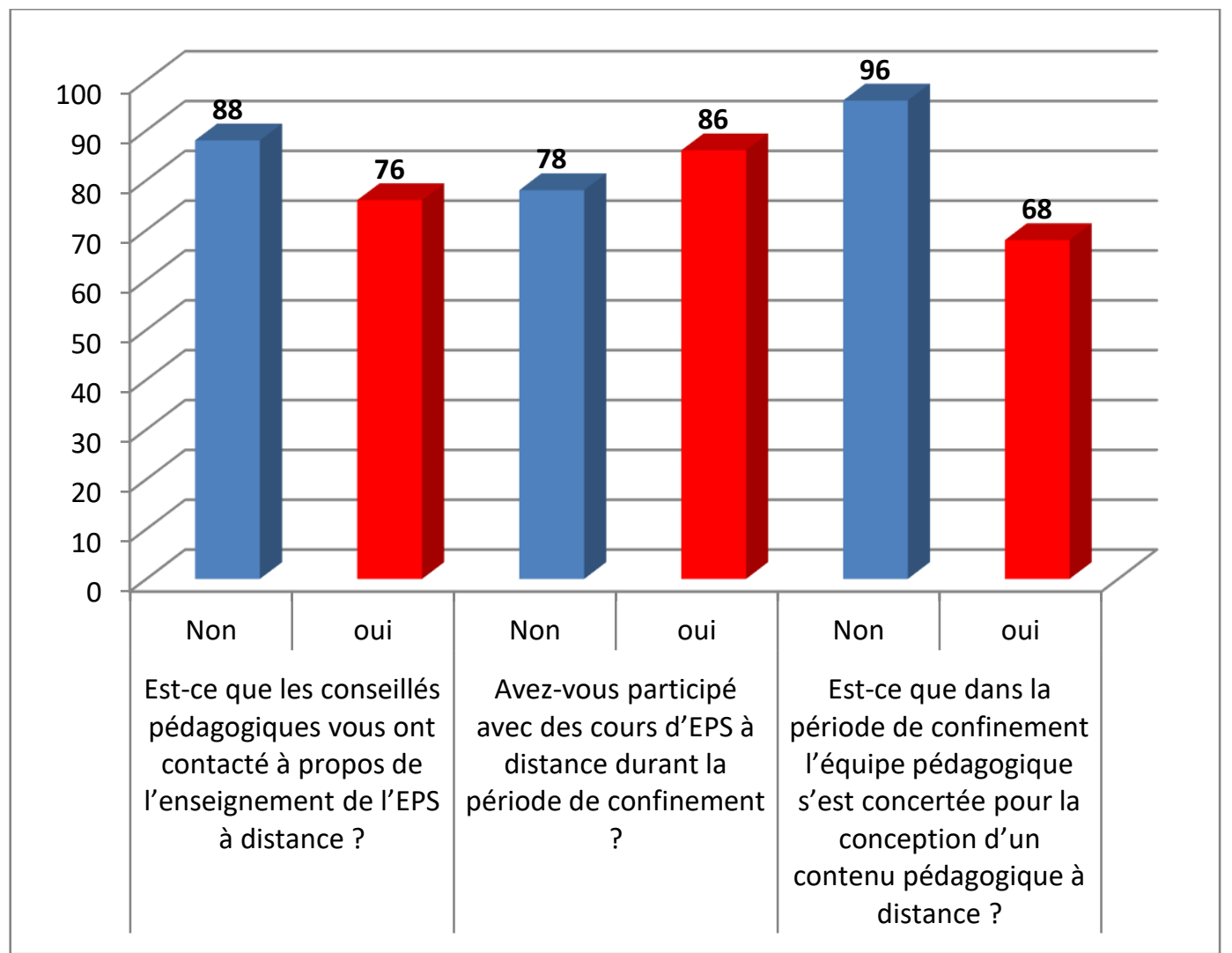

Figure 2 : Degré de communication entre les membres de l'équipe pédagogique et le corps administratif et le degré de participation avec des cours d'EPS à distance durant la période du confinement

D'après la figure 2, 88 participants soit $53.7 \%$ confirment qu'ils n'ont jamais été contactés par les conseillers pédagogiques à propos de l'enseignement à distance de l'EPS et 96 cas déclarent que l'équipe pédagogique ne s'est pas concertée pour la conception d'un contenu pédagogique à distance. C'est-à-dire qu'il y a un problème de communication au sein de l'équipe pédagogique et avec le corps administratif durant la période du confinement. Cependant, 86 participants soit $52.4 \%$ ont participé avec des cours d'EPS à distance durant la période du confinement 


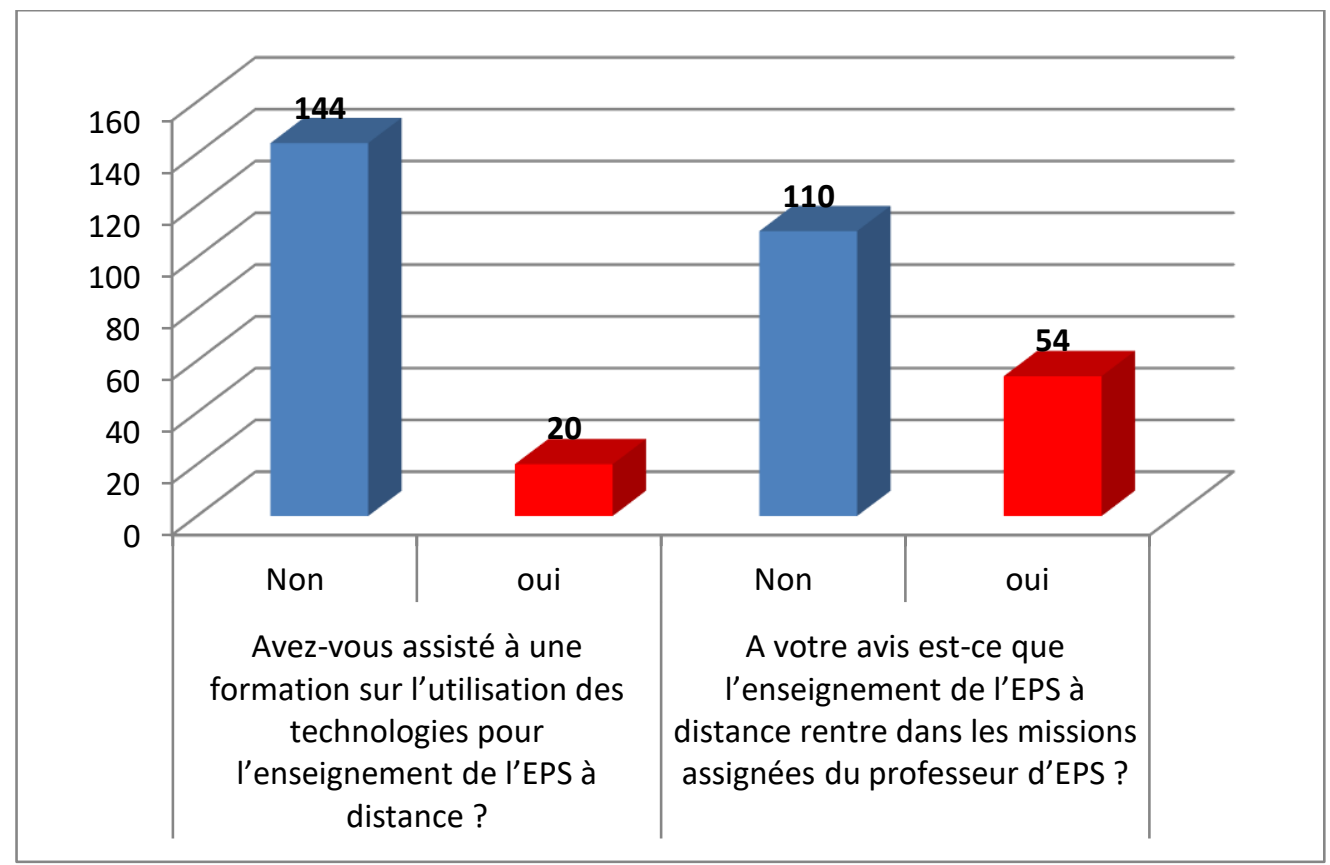

Figure 3 : Formation des participants en matière de technologie et leur mission vis-à-vis l'enseignement à distance de l'EPS

Les résultats de la figure ci-dessus montrent que 144 participants soit $87.8 \%$ n'ont pas assisté à une formation sur l'utilisation des technologies pour l'enseignement à distance de 1'EPS contre 20 cas qui ont assisté à ce type de formation, la différence est très significative $\left(\chi^{2}=93,756 ; p<0.001\right)$. D'autre part, 110 cas soit $67.1 \%$ affirment que l'enseignement à distance de l'EPS ne rentre pas dans les missions assignées au professeur d'EPS. 


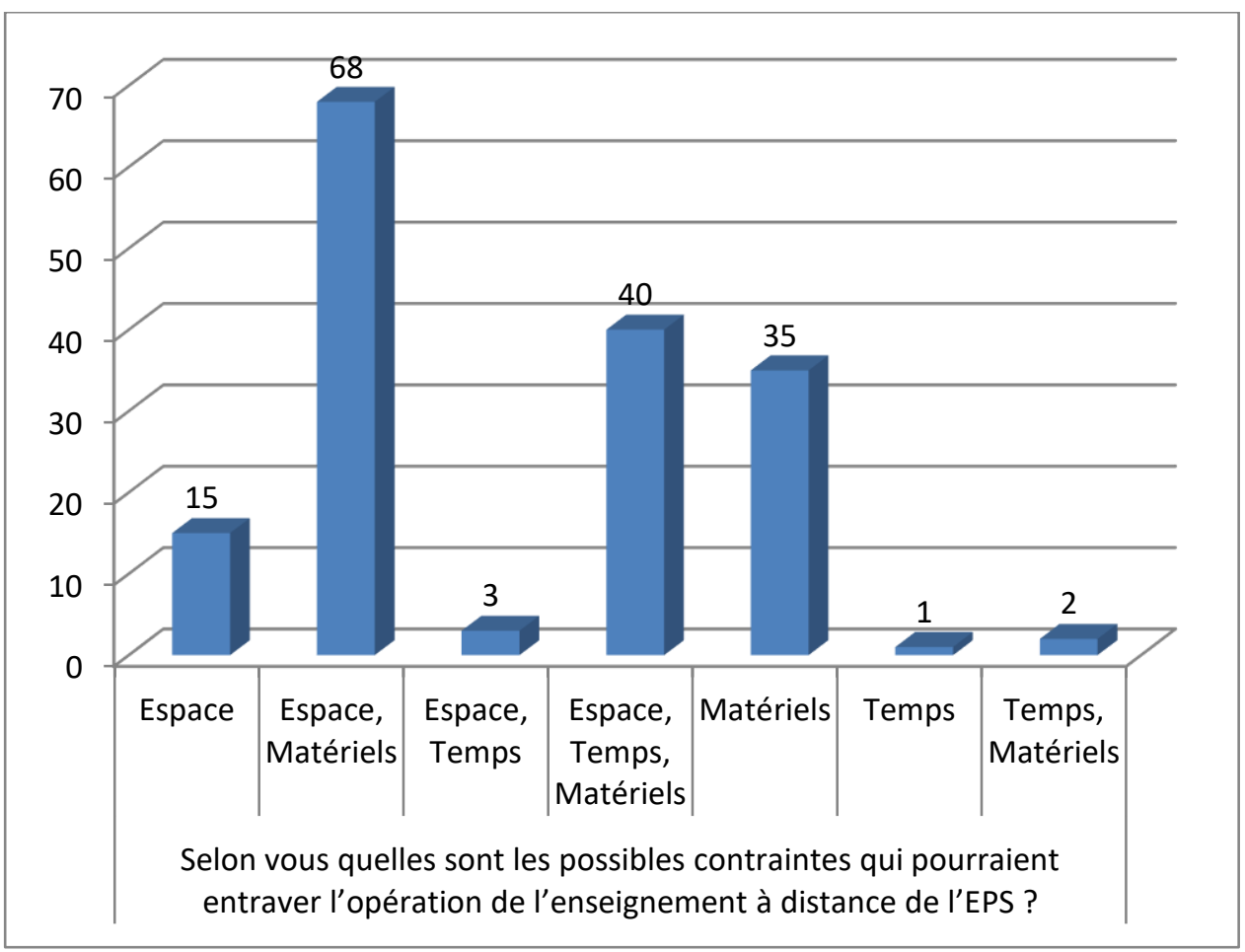

Figure4 : Les contraintes qui entravent l'opération de l'enseignement à distance de l'EPS

En ce qui concerne les contraintes qui entravent l'opération de l'enseignement à distance de l'EPS, la majorité des participants déclarent que ces contraintes sont liées principalement à l'espace, au matériel et au temps.

\section{Discussion}

L'EPS est une discipline d'enseignement à part entière qui se sert d'activités physiques pour atteindre un objectif (Hébrard, 1986). En effet, l’EPS est « une discipline entièrement à part » (Hébrard, 1998) $)^{3}$, il revient aux enseignants d'organiser leurs actions à partir de choix équilibrés, d'identifier des situations d'enseignement qui ne peuvent être uniformes, ou encore de répertorier la totalité des objectifs légitimes associés aux moyens les plus adaptés, afin de répondre aux multiples besoins des élèves

${ }^{3}$ Hébrard, A. (1998). Entretien avec Alain Hébrard. Cahiers pédagogiques, $n^{\circ} 361$, p. 9-11. 
dont ils ont la charge (Pineau \& Hébrard, 1993). D’ailleurs, il est communément admis que la mission du professeur d'EPS est de créer des situations pédagogiques à travers lesquelles il cherche de modifier le comportement moteur de l'élève. Il s'avère essentiel d'évoquer que ce mouvement initié à l'échelon national a ouvert une infinité de questions sur l'avenir de l'enseignement de l'EPS.

Du point de vue normatif, dans le référentiel métier du professeur d'EPS, ses qualifications académiques et expérientielles placent au cœur de leurs préoccupations le travail sur le terrain (le face-à-face). Le choix de l'enseignement à distance dans son optique télétravail n'est pas tout à fait en harmonie avec la fonction travail de l'enseignant d'EPS. Il est communément admis que l'enseignant d'EPS donne des consignes de travail et essaie de formaliser des critères de réussite à ses tâches motrices, de plus il apporte son soutien moral pour faciliter l'exécution des tâches pour l'apprenant. Dans l'enseignement à distance, il parait difficile de réaliser cette panoplie d'actions et d'activités.

Slagter van Tryon et Bishop (2006) avancent que parmi les faiblesses apparentes dans la présentation des cours à distances, on trouve le sentiment d'isolement des apprenants dans les cours normaux, c'est-à dire qu'en présentiel l'élève est peut être rassuré tout naturellement de son engagement moteur puisqu'il sait qu'il est accompagné par un professeur spécialisé dans l'éducation motrice, mais par contre, dans une situation d'apprentissage à distance cela va révéler un sentiment d'isolement en raison notamment du manque d'échanges éducateur-éduqué essentiel à la construction des connaissances.

L'enseignant d'EPS est nul doute maître de la conception des contenus, lorsqu'il est amené à animer un cours d'activités sportives en ligne, fort différent des activités d'enseignement en présentiel où il peut vivre une perte de repères. Ceci dit, le 
changement de posture peut conduire à un sentiment d'inconfort, voire aussi à un déséquilibre émotionnel puisque l'éducateur va penser à son identité professionnelle. L'enseignant se questionne quant au rôle qu'il exerce lorsqu'il intervient en formation à distance. Par conséquent, une sorte de résistance émerge en surface. Au regard de cette analyse, l'option enseignement de l'EPS à distance suppose un soin particulier du fait que c'est une discipline qui s'enseigne dans un espace ouvert et qui sollicite les capacités motrices et intellectuelles des scolarisés.

Apprendre à distance est différent totalement des cours en présence. Le cours à distance suppose à priori une séparation physique entre les enseignants et les apprenants. À ce titre, nous observons l'usage ultime des technologies de l'information et de la communication (TIC) (Lee, 2017). Dans cette période de transition, les situations pédagogiques d'apprentissage vont subir un changement de fond et de forme. Il s'agit bel et bien d'une nouvelle procédure de conception des contenus adaptés aux besoins des apprenants en prenant en considération leur environnement, leur niveau social, leur moyens, leur rythme, etc. En trame de fond, cette situation n'est pas habituelle pour les apprenants vis-à-vis de la matière EPS.

\section{Proposition des actions prospectives de l'enseignement de l'EPS à distance}

Dans un contexte d'innovation et de transformation des pratiques, il est temps aujourd'hui de concevoir d'autres modalités complémentaires pour l'enseignement de l'EPS. Le numérique ou le digital s'avère une opportunité idoine pour la communauté des professeurs et des inspecteurs de l'EPS. Cette situation sanitaire de covid-19 a révélé pas mal de solutions technopédagogiques utiles pour accroitre l'acquisition des apprentissages.

En s'appuyant sur les résultats de l'étude, sur nos expériences professionnelles en tant qu'ex-professeur d'EPS ainsi que de nombreux enseignements de 
l'enseignement à distance, nous essaierons de définir des pistes d'actions concrètes pour promouvoir cette approche.

Tableau 2 : Principales actions pour promouvoir l'enseignement à distance de l'EPS

\begin{tabular}{|c|c|}
\hline Les axes prioritaires & Pistes d'actions \\
\hline $\begin{array}{l}\text { Formation et } \\
\text { développement des } \\
\text { compétences }\end{array}$ & $\begin{array}{l}\text { Engager les professeurs d'EPS et les inspecteurs de l'EPS dans un processus de } \\
\text { développement des compétences; } \\
\text { Concevoir un projet d'accompagnement pour les professeurs manquant de } \\
\text { compétences technologiques, et offrir un suivi individuel ; } \\
\text { S'intéresser aux représentations des professeurs sur l'usage des outils numériques et } \\
\text { digitaux } \\
\text { Former les enseignants sur les outils technologiques et les supports médiatiques à } \\
\text { exploiter. }\end{array}$ \\
\hline $\begin{array}{l}\text { Conception et } \\
\text { réalisation des contenus }\end{array}$ & $\begin{array}{l}\text { Engagement des inspecteurs de l'EPS dans la définition des thématiques } \\
\text { d'apprentissage à distance; } \\
\text { Contribution des inspecteurs de l'EPS à offrir les ressources numériques disponibles } \\
\text { pour optimiser la qualité de réception et d'acquisition des apprentissages; } \\
\text { Les inspecteurs de l'EPS sont amenés à guider les activités d'enseignement à distance et } \\
\text { conseiller sur l'exploitation des technologies et l'utilisation des environnements } \\
\text { numériques d'apprentissage ; } \\
\text { Proposition aux enseignants d'EPS des scénarios pédagogiques adaptés aux conditions } \\
\text { sociales des apprenants; } \\
\text { Cibler le « point focal » du professeur, ses préférences d'investissement, ses } \\
\text { compétences conceptuelles et procédurales. } \\
\text { Approcher l'opération d'enseignement à distance avec habileté et offrir des voies } \\
\text { facultatives. }\end{array}$ \\
\hline
\end{tabular}




\begin{tabular}{|c|c|}
\hline $\begin{array}{l}\text { Communication entre } \\
\text { les acteurs }\end{array}$ & $\begin{array}{l}\text { Etablir un rapport de communication fluide et direct entre le corps professoral d'une } \\
\text { part et d'autre part avec les inspecteurs de l'EPS sur les différents types de supports } \\
\text { médiatiques disponibles (zoom, Microsoft-teams, google meet, les applications de } \\
\text { chat ; } \\
\text { Promouvoir et reconnaitre l'action des unités pédagogiques pour la conception et la } \\
\text { réalisation des contenus technopédagogiques; } \\
\text { Favoriser un dialogue constant et bidirectionnel entre les enseignants et les } \\
\text { inspecteurs; } \\
\text { Savoir communiquer sur la valeur ajoutée de l'enseignement à distance; } \\
\text { Repérer les problèmes rencontrés dans l'opérationnalisation des actions et envisager } \\
\text { des réunions de guidage et de soutien; } \\
\text { Identifier la capacité d'engagement des enseignants dans la réalisation des actions } \\
\text { d'enseignement à distance. }\end{array}$ \\
\hline Centre de formation & $\begin{array}{l}\text { Accroitre le nombre d'heures dans les modules dédiés pour les Technologies de } \\
\text { l'information et de la communication (TIC) que ce soit pour les inspecteurs ou les } \\
\text { professeurs en formation } \\
\text { Contribution des enseignants formés à concevoir des contenus et des scénarios } \\
\text { technopédagogiques } \\
\text { Contribution des acteurs (professeurs d'EPS, les inspecteurs et les académiciens) à la } \\
\text { conception d'un référentiel dédié à l'enseignement de l'EPS à distance. }\end{array}$ \\
\hline
\end{tabular}

\section{Conclusion}

Personne n'a songé qu'un jour l'enseignement de l'EPS à distance par n'importe quelle forme sera transité dans l'espace numérique. Cette contribution propose donc les premières briques d'un nouveau champ de recherche. L'enseignement de l'EPS peut dans certaines conditions possibles devenir une discipline hybride qui rallie une activité 
d'enseignement en présentiel et en enseignement à distance. Relativement à l'enseignement à distance une palette de choix s'affiche en éclair. Pour bien préciser ce point les enseignants d'EPS sont les metteurs en scène de cette nouvelle approche. Il s'agit donc de réfléchir sur les thématiques, les mécanismes de fonctionnement, les ressources disponibles et les compétences sollicitées afin de concrétiser cette nouvelle procédure pédagogique dans l'enseignement de l'EPS.

\section{Références}

Blandin, B. (1990). Formateurs et formation multimédia : les métiers, les fonctions, l'ingénierie. Les Éditions d'organisation.

Jaillet, A. (2005). Peut-on repérer les effets de l'apprentissage collaboratif à distance ? Distances et savoirs, 3(1), 49-66.

OQLF : www.granddictionnaire.com

Charnet, C. (2019). Comment réaliser une formation ou un enseignement numérique à distance ? De Boeck Supérieur.

https://ec.europa.eu/transparency/regdoc/rep/1/2001/FR/1-2001-172-FR-F1-1.Pdf consulté le 18/05/2020

Romiszowski, A. (2003). The future of E-learning as an educational innovation : Factors influencing project success and failure. RevistaBrasileira de AprendizagemAberta e a Distância, 2.

Joab, M., \& Dumont, B. (2008, May). E-Quality : Mise en œuvre de la qualité pour la FOAD dans l'enseignement supérieur en Europe. In AIPU'08 : 25e Congrès de l'Association Internationale de Pédagogie Universitaire (p. 10).

Dumont, C. (2007). Formation des enseignants. Dans D. Boeck (Éd.), L'enseignement en ligne à l'université et dans les formations professionnelles. (Pp. 359). Bruxelles.

Gélis, J. M. (2013). L'engagement des enseignants dans un dispositif d'enseignement à distance. Distances et médiations des savoirs Page consultée le 15 mai 2013 de http://dms.revues.org/175.

George, S., Prévôt, P., Amghar, Y., \& Pierson, J. M. (2004, November). Complexité des situations pédagogiques e-learning dans un contexte multi-culturel, collaboratif et synchrone. In Conférence TICE Méditerranée. 
Denis, B., Watland, P., Pirotte, S., Verday, N. (2004), Roles and Competencies of the eTutor (Learn Nett project), Networked Learning Conference 2004, 5 - 7 april 2004

Stockless, A, et Villeneuve, S. (2017). Développer ses compétences numériques : doiton devenir un expert? Dans Romero, M. et al (dir.), Usages créatifs du numérique pour l'apprentissage au XXIe siècle (141-148). Québec, QC : PUQ.

Audet, L. et Richer, M. (2014). Tuteurs, Apprenants, Enseignants. Similitudes et différences, constantes et évolution des compétences pour la formation à distance. Communication, Colloque de l'ACFAS, Montréal, QC.

Parr, M. (2019). Pour apprivoiser la distance : guide de formation et de soutien aux acteurs de la formation à distance.

http://www.inrs.fr/risques/teletravail-situation-exceptionnelle/prevenir-comportementsedentaire.html (consulté le 18/05/2020).

Le Dr François Carré confirme que « Toutes les études scientifiques ont prouvé que l'activité physique modérée boostait » notre système immunitaire.

Slagter van Tryon, P. J., Bishop, J. M. (2006). Identifying e-mmediacy strategies for Web-based instruction. Quarterly Review of Distance Education, 7 (1), 49-62.

Hébrard, A. (1986). L'éducation physique et sportive. Réflexions et perspectives. Revue STAPS et Editions « Revue E. P. S. », Paris, 271 p.

Hébrard, A. (1998). Entretien avec Alain Hébrard. Cahiers pédagogiques, n³61, p. 911.

Pineau, C., \& Hébrard, A. (1993). L'EPS, aujourd'hui. Revue EPS, 240.

Lee, K. (2017). Rethinking the accessibility of online higher education : A historical review. The Internet and Higher Education, 33, 15-23. doi:10.1016/j.iheduc.2017.01.001

Parr, M. (2019). Pour apprivoiser la distance : guide de formation et de soutien aux acteurs de la formation à distance. 